\title{
Non-specific immunological effects of selected routine childhood immunisations: systematic review
}

\author{
Rama Kandasamy, 1,2 Merryn Voysey, 1,2,3 Fiona McQuaid,, 1,2 Karlijn de Nie,, Rebecca Ryan,,,2 \\ Olivia Orr, ${ }^{1,2}$ Ulrike Uhlig, ${ }^{4}$ Charles Sande, ${ }^{1,2}$ Daniel O'Connor, ${ }^{1,2}$ Andrew J Pollard ${ }^{1,2}$
}

${ }^{1}$ Oxford Vaccine Group, Department of Paediatrics, University of Oxford, Churchill Hospital, Oxford OX3 7LE, UK ${ }^{2}$ NIHR Oxford Biomedical Research Centre, Oxford, OX3 7LE, UK

${ }^{3}$ Nuffield Department of Primary Care Health Sciences, University of Oxford, Oxford, OX2 6GG, UK

${ }^{4}$ Department of Paediatrics, Children's Hospital Oxford,

Oxford University Hospitals NHS

Trust, Oxford, OX3 9DU, UK

Correspondence to: R Kandasamy rama.kandasamy@paediatrics. ox.ac.uk

Additional material is published online only. To view please visit the journal online.

Cite this as: $B M / 2016 ; 355: 15225$ http://dx.doi.org/10.1136/bmj.i5225

Accepted: 20 September 2016

\section{ABSTRACT}

OBJECTIVE

To identify and characterise non-specific

immunological effects after routine childhood vaccines against BCG, measles, diphtheria, pertussis, and tetanus.

\section{DESIGN}

Systematic review of randomised controlled trials, cohort studies, and case-control studies.

\section{DATA SOURCES}

Embase, PubMed, Cochrane library, and Trip searched between 1947 and January 2014. Publications submitted by a panel of experts in the specialty were also included.

\section{ELIGIBILITY CRITERIA FOR SELECTING STUDIES}

All human studies reporting non-specific immunological effects after vaccination with standard childhood immunisations. Studies using recombinant vaccines, no vaccine at all, or reporting only vaccine specific outcomes were excluded. The primary aim was to systematically identify, assemble, and review all available studies and data on the possible nonspecific or heterologous immunological effects of BCG; measles; mumps, measles, and rubella (MMR); diphtheria; tetanus; and pertussis vaccines.

RESULTS

The initial search yielded 11168 references; 77 manuscripts met the inclusion criteria for data analysis. In most included studies (48\%) BCG was the vaccine intervention. The final time point of outcome measurement was primarily performed (70\%) between one and 12 months after vaccination. There was a high risk of bias in the included studies, with no single study rated low risk across all assessment criteria. A total of 143 different immunological variables were reported, which, in conjunction with differences in

\section{WHAT IS ALREADY KNOWN ON THIS TOPIC}

Observational studies across a limited geographical distribution have suggested the presence of non-specific effects on all cause mortality for BCG, measles, diphtheria, pertussis, and tetanus vaccines

No causal immunological mechanism has yet been elucidated

\section{WHAT THIS STUDY ADDS}

In some BCG and measles vaccine studies, there are consistent trends or patterns of immunological response after vaccination that are suggestive of non-specific immunological effects

There is no conclusive immunological evidence from previously conducted human studies to support the presence of clinically relevant, geographically generalisable, non-specific immunological effects after vaccination with BCG, diphtheria, pertussis, tetanus, or measles containing vaccines measurement units and summary statistics, created a high number of combinations thus precluding any meta-analysis. Studies that compared BCG vaccinated with unvaccinated groups showed a trend towards increased IFN- $\gamma$ production in vitro in the vaccinated groups. Increases were also observed for IFN- $\gamma$ measured after BCG vaccination in response to in vitro stimulation with microbial antigens from Candida albicans, tetanus toxoid, Staphylococcus aureas, lipopolysaccharide, and hepatitis B. Cohort studies of measles vaccination showed an increase in lymphoproliferation to microbial antigens from tetanus toxoid and $C$ albicans. Increases in immunogenicity to heterologous antigens were noted after diphtheriatetanus (herpes simplex virus and polio antibody titres) and diphtheria-tetanus-pertussis (pneumococcus serotype 14 and polio neutralising responses) vaccination.

\section{CONCLUSIONS}

The papers reporting non-specific immunological effects had heterogeneous study designs and could not be conventionally meta-analysed, providing a low level of evidence quality. Some studies, such as BCG vaccine studies examining in vitro IFN- $\gamma$ responses and measles vaccine studies examining lymphoproliferation to microbial antigen stimulation, showed a consistent direction of effect suggestive of non-specific immunological effects. The quality of the evidence, however, does not provide confidence in the nature, magnitude, or timing of non-specific immunological effects after vaccination with BCG, diphtheria, pertussis, tetanus, or measles containing vaccines nor the clinical importance of the findings.

\section{Introduction}

Many published reports and commentaries have suggested that several vaccines routinely administered to infants could have heterologous or non-specific effects on mortality, unrelated to the prevention of illness and deaths caused by the specific diseases against which the vaccines were developed..$^{1-3}$ For example, studies have suggested that receipt of both the BCG and measles vaccine are associated with a reduced risk of death (that is, all cause mortality) beyond that expected by a reduction in deaths from measles and tuberculosis, while receipt of diphtheria-tetanus-pertussis (DTP) vaccine might be associated with an increased risk of death, at least among female infants. ${ }^{4-6}$ Nearly all studies that showed these effects were observational or derived from secondary analyses. Consequently, poorly controlled or uncontrolled confounding and various types of selection and information bias have been suggested as alternative justifications for these findings. ${ }^{78}$ 
The biological plausibility of one or more vaccines having heterologous effects, either detrimental or beneficial, is supported by several studies in animals and observations in humans.9-12 Indeed it is these heterologous properties that are exploited in specific circumstances in adults in whom BCG has been used for the treatment of bladder cancer and melanoma, while MMR has been used as a treatment for warts. ${ }^{13-15}$ Nevertheless, the biological mechanisms and immune pathways that could underlie and rationalise such effects remain largely unspecified. The WHO Strategic Advisory Group of Experts (SAGE) requested the WHO Secretariat to review the evidence surrounding the possible non-specific/heterologous effects of vaccines included in the routine infant immunisation schedule in 2013. ${ }^{16}$ The WHO Secretariat working group commissioned this review to determine whether the current evidence is sufficient to warrant further scientific investigation and, if so, to define the path towards obtaining unequivocal evidence on these issues that would support future robust, evidence based adjustments to immunisation policies, if warranted.

The possible implications of any such heterologous effects of vaccines for the formulation or re-formulation of the infant immunisation schedule remain unclear, but it has been suggested that if such effects can be established beyond a reasonable doubt, the infant immunisation schedule might need to be reconfigured. ${ }^{16}$ Previous reviews, including periodic assessments by the WHO Global Advisory Committee on Vaccine Safety, have indicated that any such effects remain inconclusive and are therefore not a justification for altering the current schedule recommendations. ${ }^{17}$ At the meeting of SAGE during which the data from this review were presented, no changes to the current policy were recommended. ${ }^{18}$

We systematically identified, assemble, reviewed, and critically appraised all available human studies with immunological endpoints describing the possible non-specific or heterologous effects of BCG, diphtheria, pertussis, tetanus, and measles containing vaccines.

\section{Methods \\ Definitions}

We defined specific immunological effects as an effect of immunisation on an immunological variable in response to an antigen contained within the initial immunisation. The terms heterologous and nontargeted effects have also been used in the literature to describe non-specific immunological effects. For the purpose of this review we defined non-specific immunological effects as an effect on the immune system as a result of immunisation that modifies the way it subsequently responds to antigens that were not present in the initial immunisation.

\section{Study design and selection criteria}

Using a comprehensive search strategy, we identified and critically appraised available evidence (published and unpublished) that addressed possible non-specific effects of vaccines. We included in the review randomised controlled trials, quasi-randomised control trials, clinical trials, cohort studies, case-control studies, case series, and case reports. The vaccines examined included live attenuated vaccines (BCG and measles containing vaccines), inactivated vaccines, and toxoids (all diphtheria and tetanus toxoids, and Bordetella pertussis containing vaccines). Though the target population was infants aged under 5 , inclusion of studies was not limited to this age group to ensure all relevant studies were identified. Sex, age at vaccination, and co-administration of vitamin A were examined as possible effect measure modifiers.

Were excluded ecological, animal, and in vitro studies; studies using recombinant vaccines or no vaccine at all; and those studies reporting/generating only immunological endpoints specific to the study vaccine.

\section{Search strategy}

Embase.com, which includes all records from Medline, was searched from 1947 to December 2012 (appendix 1). Complementary, less extensive searches of the PubMed library, the Cochrane library, and trip database, were performed to detect any articles missed by the search on Embase.com. In addition, we manually searched the reference lists of all included articles found and all relevant review articles to identify studies not included in the previously described search. Experts in the specialty were asked if they were aware of any unpublished reports of studies possibly meeting the inclusion criteria. Full text of all articles identified were sought, using internet downloads, interlibrary loans, and contacting of authors. Articles in any language were sought. We carried out a further limited search from December 2012 to January 2014 in the PubMed library using the same search terms to provide an update. Experts in the specialty were also asked to review the initial search results and identify any further studies that could be included.

\section{Data extraction}

We used specifically created data extraction forms and DistillerSR software to acquire consistent data from studies, such as participants, methods, potential confounders and background data. All relevant data were extracted from articles meeting inclusion criteria and entered on the database. There was no pre-specified effect measure that was of interest but rather all summary estimates of effect were extracted whenever possible.

\section{Selection of eligible Studies}

Two independent reviewers examined each full text article, and a database of studies considered eligible for inclusion was created (tables A-F in appendix 2). We included in the review studies identified by both reviewers as being eligible for inclusion and having adequate data for extraction. Authors of studies in which non-specific immunological data were generated but not reported were requested to provide data. When there were discrepancies, the reasons for these were discussed, and a decision about inclusion was reached 
by consensus. If there was no agreement, a further independent reviewer adjudicated to make a final decision regarding eligibility.

\section{Assessment of risk of bias}

At least two independent reviewers assessed each included study according to the Cochrane Library risk of bias tool (tables G-L, appendix 2). When there was a conflict in the assessment, a third independent reviewer adjudicated to make a final decision. The overall risk of bias was calculated by assigning each criteria a score of 0 for high, 1 for unclear, and 2 for low and then averaging the total score across all of the criteria.

\section{Data analysis}

We generated descriptive tables summarising information about study design, study quality, and results of all included studies. Data on non-specific immunological effects were extracted from papers, which reported summary statistics in tabular form. When results were presented in figures, we extracted data whenever possible with GetData Graph Digitizer version 2.26.0.20.

We summarised the overall immunological outcomes of the included studies graphically for all available variables to provide a perspective on the effect of vaccination on these variables. We calculated the direction of effect for each variable by creating a ratio of the response in those vaccinated compared with the response in the unvaccinated participants, or alternatively the ratio of the estimated response after vaccination compared with the response before vaccination. The response could be presented as a median, mean, geometric mean, fold-rise, or proportion of seroconversion, depending on the statistics reported in the paper. Participants were not all measured at comparable time points across studies nor were of similar age. We could not formally combine these ratios as they are statistically non-comparable, the timings differ, and many studies did not present estimates of variability. Not all studies reported results of significance tests and for those that did the statistical test reported was not always the appropriate one. Furthermore, because of the multitude of variables tested and small study sizes, there were related multiple testing issues that would have increased the rate of type 1 errors. For these reasons we designed the plots to be descriptive of the overall diversity of responses and point to any general trends that could be occurring in the data without the calculation of any summary statistics or testing specific hypotheses that would have required more consistent data. For papers that reported comparisons at multiple time points for the same children we plotted only the first comparison so that each cohort of children is reported only once per study per variable per type of stimulant.

\section{Patient involvement}

No patients were involved in setting the research question or the outcome measures, nor were they involved in the design and implementation of the study. There are no plans to involve patients in dissemination.

\section{Results}

The search yielded 11168 references, and 77 studies met our eligibility criteria (fig 1). Fourteen of these studies were from submissions made by experts in the specialty. Of the two studies published on the randomised controlled trial by Burl and colleagues, we included additional data from the associated $\mathrm{PhD}$ thesis. ${ }^{19}$ We identified relatively equal proportions of randomised controlled trials, cohort studies, and case-control studies (table 1). There was a wide range (3-2345; mean 206) of total study participants involved across the studies. Most studies (37/77, 48\%) used BCG as the study vaccine intervention, while 47/77 (61\%) were exclusively conducted in children. In 54/77 (70\%) studies, the final time point of outcome measurement was performed between one and 12 months after vaccination. At least one non-specific immunological variable was reported as significant in 29/77 (38\%) of the studies (tables 2-5). Because of the heterogeneity, we could not conduct a meta-analysis and for that reason none of the trends, where evident, are statistically sound.

\section{BCG vaccine studies}

Overall 37 studies were identified that measured non-specific immunological effects of BCG vaccination (appendix 2). In 11 of these papers, the results of assays conducted were not reported as they were not the main focus of the paper. Of the included studies, 24 enrolled children aged under 5 years. Twenty papers reported

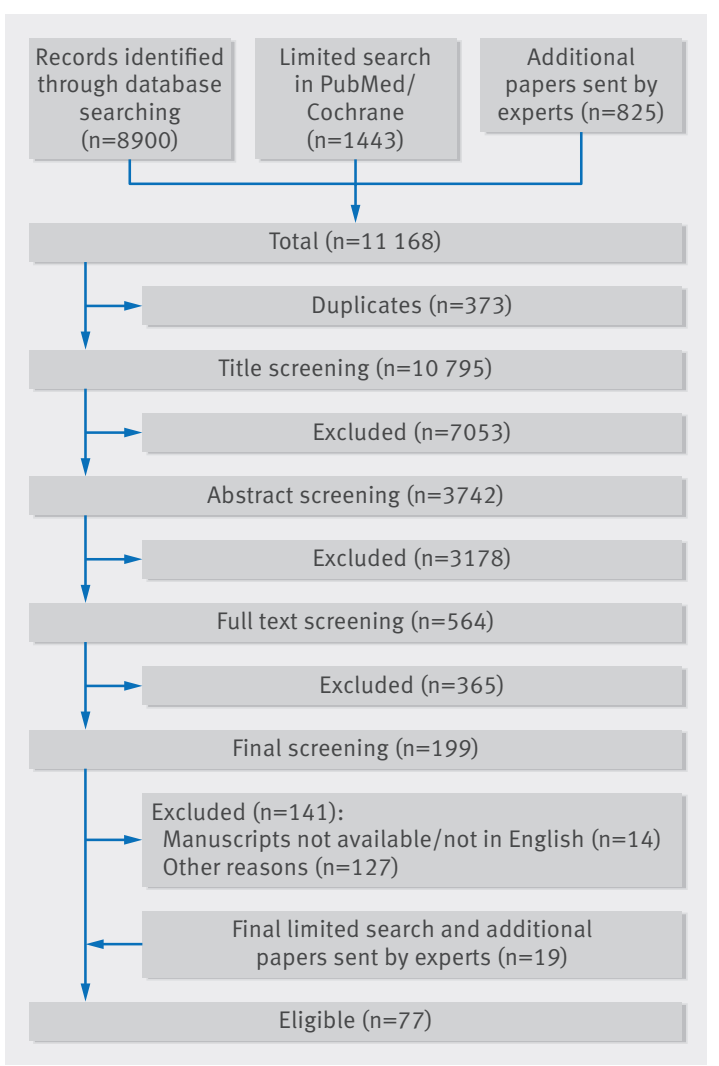

Fig 1 | Identification of studies in review of non-specific immunological effects of selected routine childhood immunisations 


\begin{tabular}{|c|c|}
\hline Study vaccine & No of participants \\
\hline BCG & 37 \\
\hline Measles & 14 \\
\hline MMR & 3 \\
\hline DTP & 7 \\
\hline Pertussis & 1 \\
\hline DT & 4 \\
\hline TT & 11 \\
\hline \multicolumn{2}{|l|}{ Other vaccine/s used in study: } \\
\hline Yes & 24 \\
\hline No & 31 \\
\hline Not described/not applicable & 22 \\
\hline \multicolumn{2}{|l|}{ Age group: } \\
\hline Neonate & 15 \\
\hline Infant & 18 \\
\hline Children & 14 \\
\hline Adults & 19 \\
\hline Elderly & 0 \\
\hline Combination & 11 \\
\hline \multicolumn{2}{|l|}{ Sex of study population: } \\
\hline Male and female & 39 \\
\hline Male & 2 \\
\hline Female & 1 \\
\hline NR & 35 \\
\hline \multicolumn{2}{|l|}{ Geographical location: } \\
\hline Africa & 19 \\
\hline Europe & 22 \\
\hline Asia & 8 \\
\hline Americas & 20 \\
\hline Oceania & 4 \\
\hline Combination & 4 \\
\hline \multicolumn{2}{|l|}{ Co-administration with vitamin $\mathrm{A}$ ? } \\
\hline Yes & 3 \\
\hline No/NR & 74 \\
\hline \multicolumn{2}{|c|}{ Presence of attribute that could affect response? } \\
\hline Yes & 22 \\
\hline No & 55 \\
\hline \multicolumn{2}{|c|}{ Interval between vaccine administration and final outcome measure (months): } \\
\hline$<1$ & 11 \\
\hline $1-<6$ & 29 \\
\hline $6-\leq 12$ & 25 \\
\hline$>12$ & 10 \\
\hline NR & 2 \\
\hline \multicolumn{2}{|l|}{ No of participants: } \\
\hline Mean & 206 \\
\hline Median & 77 \\
\hline Range & $3-2345$ \\
\hline \multicolumn{2}{|l|}{ Study design: } \\
\hline Randomised controlled trial & 25 \\
\hline Prospective cohort & 23 \\
\hline Prospective case-control & 23 \\
\hline Other & 6 \\
\hline
\end{tabular}

non-specific immunological effects with data reported in tables or graphs, which could be extracted with a digitizer program, and one study supplied raw data. These papers reported 89 different immunological variables. There were 20 types of stimulants used in theses assays, resulting in 167 unique combinations.

Immunological responses from unstimulated and phytohaemagglutinin (PHA) stimulated cultures were most commonly reported (fig 2; appendix 4 provides detailed tabulated data for the figure). No general patterns according to pro-inflammatory or antiinflammatory classifications were observable for unstimulated responses when comparisons were between vaccinated and unvaccinated groups, though a distinct increase in IFN- $\gamma$ was noted within one cohort study reporting cytokine responses in unstimulated cultures after BCG vaccination. ${ }^{20}$

Cytokine responses in PHA stimulated cultures showed a trend towards increases in vaccinated compared with unvaccinated groups for all cytokines, although there were studies assessing the pro-inflammatory cytokines IFN- $\gamma$ and TNF- $\alpha$ that also fell below the null value. In comparisons of cytokine responses in PHA stimulated cultures before and after vaccination within a cohort study, there was a trend towards an increase in pro-inflammatory cytokines and a decrease in anti-inflammatory cytokines.

IFN- $\gamma$ was the most commonly reported immunological variable. Data for IFN- $\gamma$ were extracted from 11 papers and one PhD thesis and one study author supplied unpublished raw data from unstimulated assays on request (fig 2). Those studies that made comparisons between vaccinated and unvaccinated groups showed a trend towards an increase in IFN- $\gamma$ after stimulation. Increases after vaccination were observed in a small cohort study of IFN- $\gamma$ measured in response to in vitro stimulation with Candida albicans and Staphylococcus aureas. ${ }^{22}$

$\mathrm{T}$ cells and $\mathrm{T}$ cell subsets were the most commonly reported leukocyte variable (fig 2). Ex vivo total leukocyte counts had larger cohort sizes and trended around the null value. Counts of subsets for vaccinated compared with unvaccinated groups showed a decrease in neutrophils, CD8, and $\gamma \delta^{+}$T cells; increase in CD4 T cells and monocytes; and no consistent direction for eosinophils. Cohort studies that compared values before and after vaccination showed an increase in total lymphocytes and $\mathrm{CD}_{14^{+}}$cells, decrease in $\alpha \beta^{+} \mathrm{T}$ cells, and inconsistent effect for $\gamma \delta^{+}$T cells. ${ }^{22} 26$ In vitro proliferation assays to tetanus toxoid and hepatitis B surface antigen (HBsAg) all showed increases in the vaccinated compared with unvaccinated group..$^{25} 48$

\section{Measles vaccine studies}

Various different measles vaccine strains and titres were used, with the Edmonston-Zagreb and Schwarz strains most commonly applied (appendix 2, table B). Data available from measles studies reported 23 different immunological variables including $B$ cells, $\beta 2$ microglobin, CD4, CD4:CD8 ratio, CD8, IFN- $\gamma$, IL-10, IL-2, sIL-2Ra, IL-4, IL-6, lymphocytes, lymphoproliferation, malaria parasites, MIP-1 $\beta$, Neopterin, sCD4, sCD8, T-cell proliferation, TNF- $\alpha$, and total white blood cell count (WBC). There were six types of stimulants used in these assays (including $C$ albicans, PHA, and tetanus toxoid), resulting in 31 unique combinations. All the papers contained children aged under 5 .

In studies of PHA stimulated assays IFN- $\gamma$ was the most commonly reported variable followed by IL-10 (fig 3; 


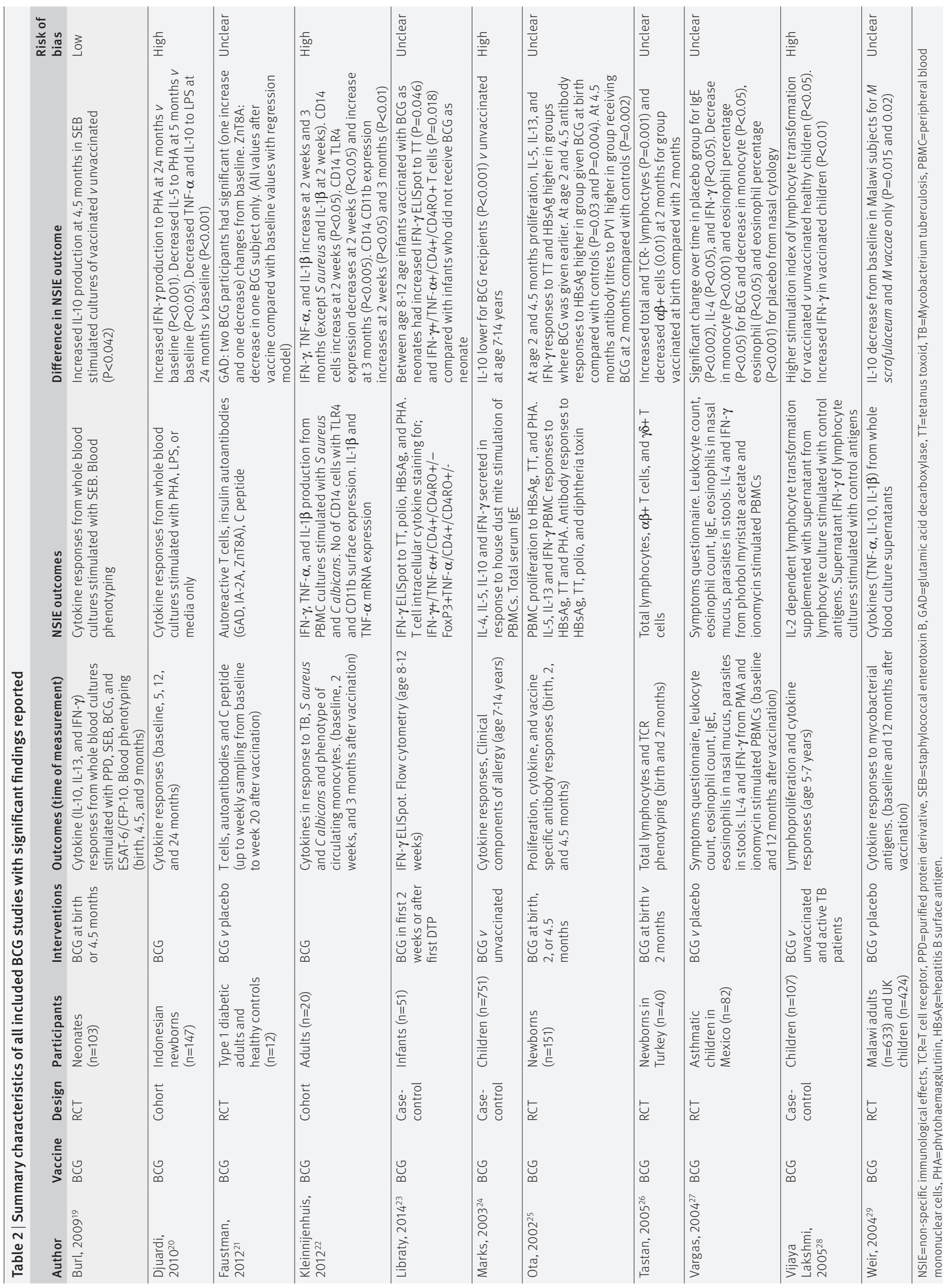




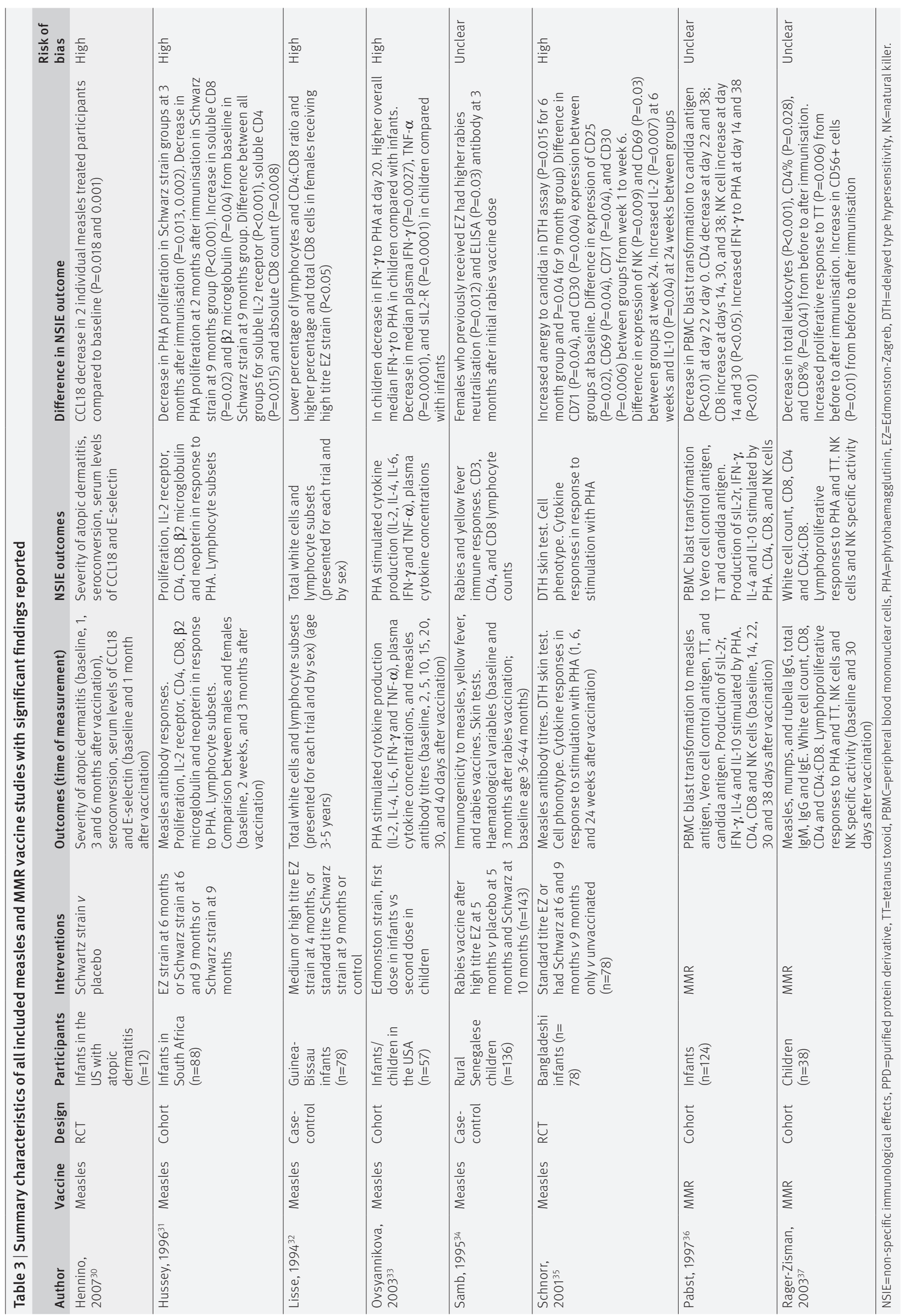




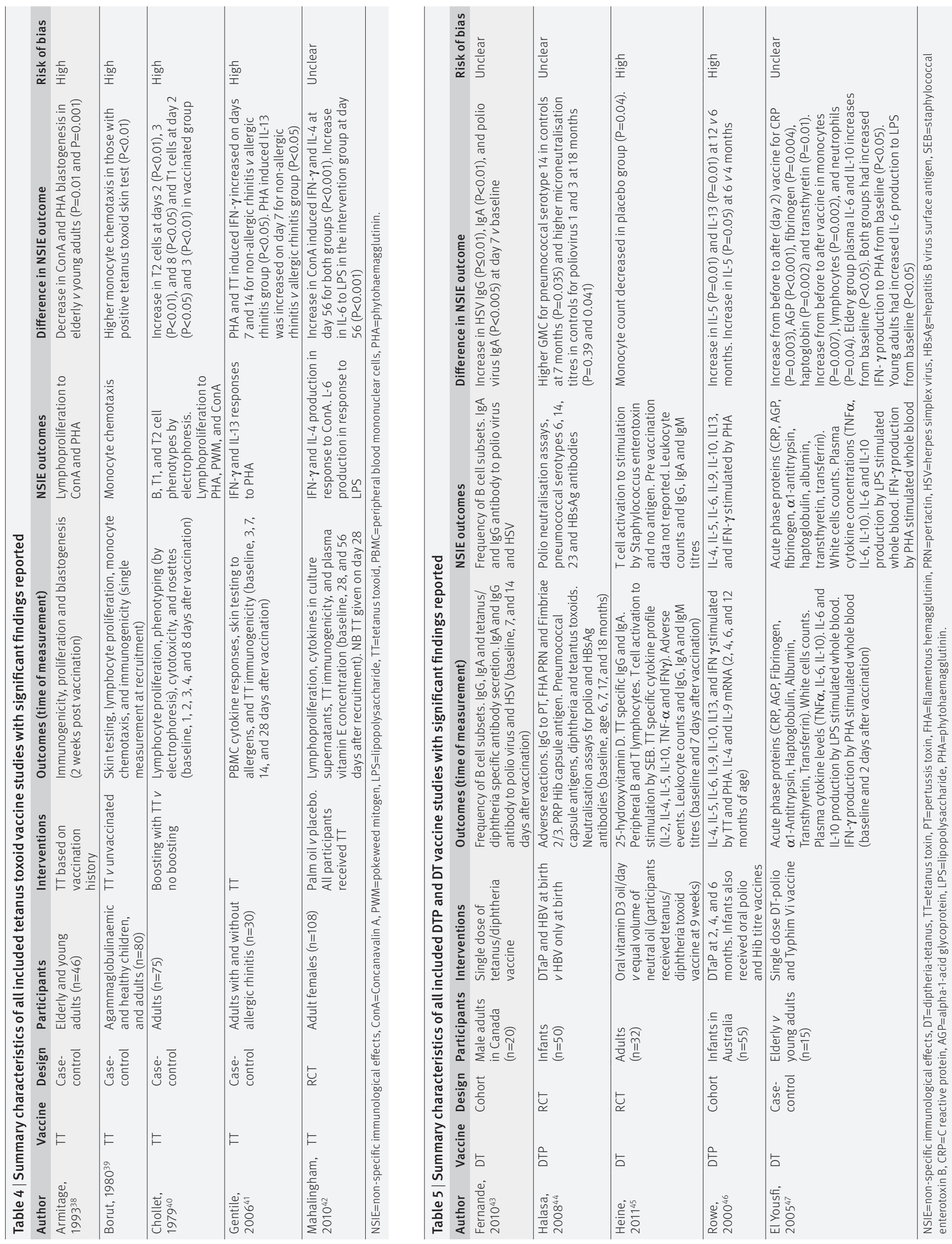

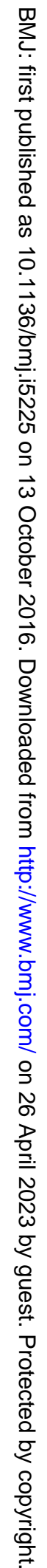


Ratios of geometric means $\bigcirc$ Ratios of medians (1) Ratios of means

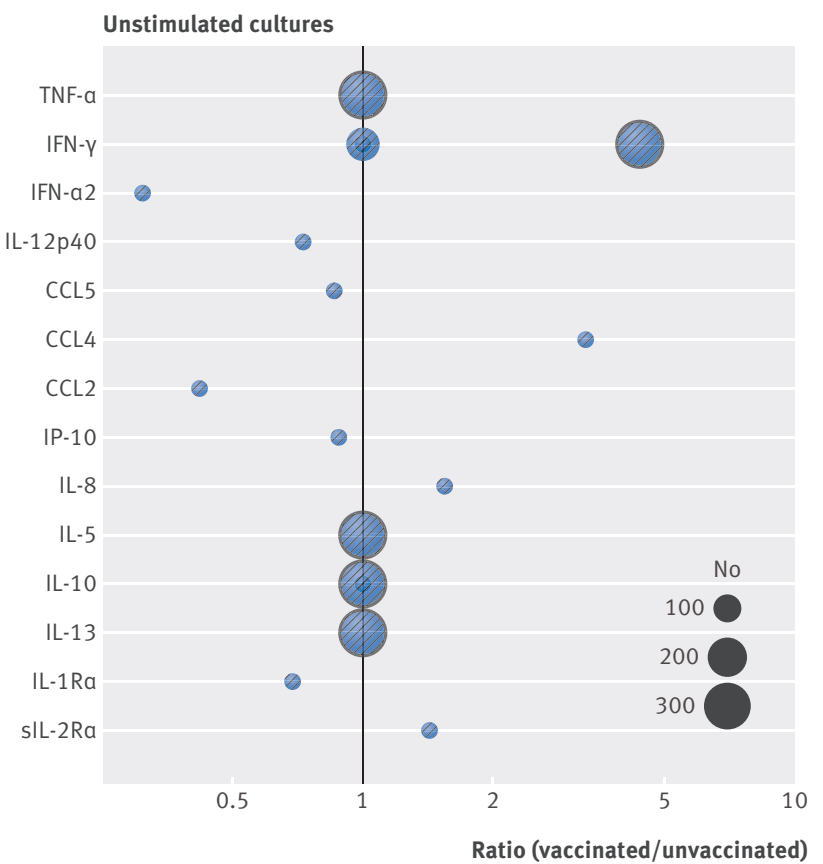

IFN-y responses

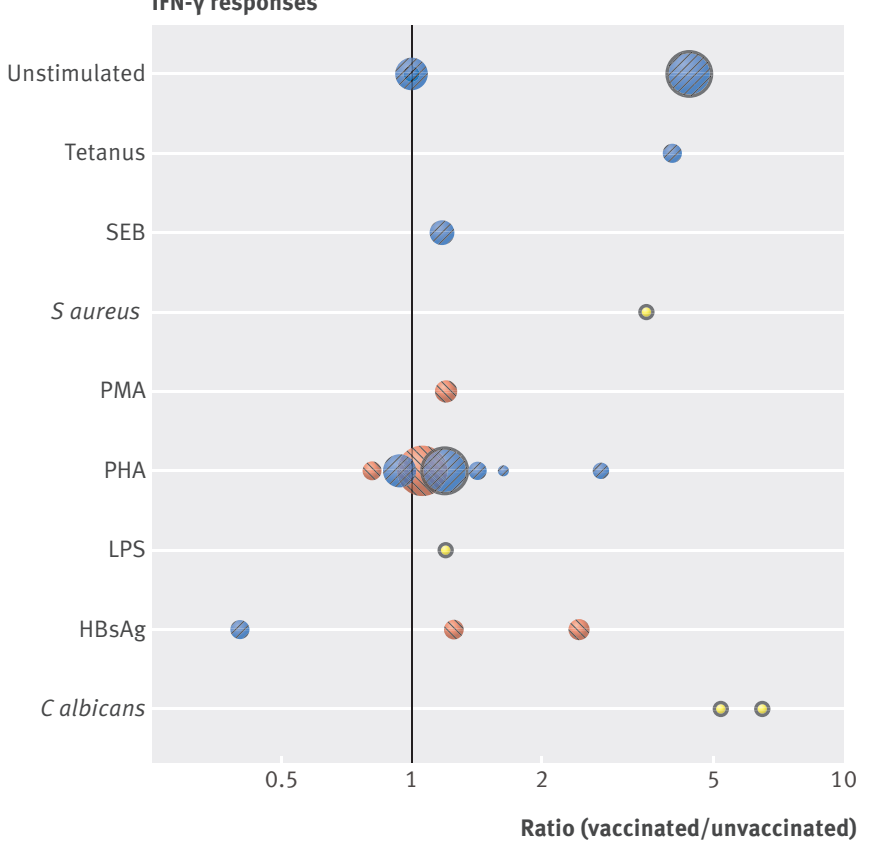

Mean within-person fold rise

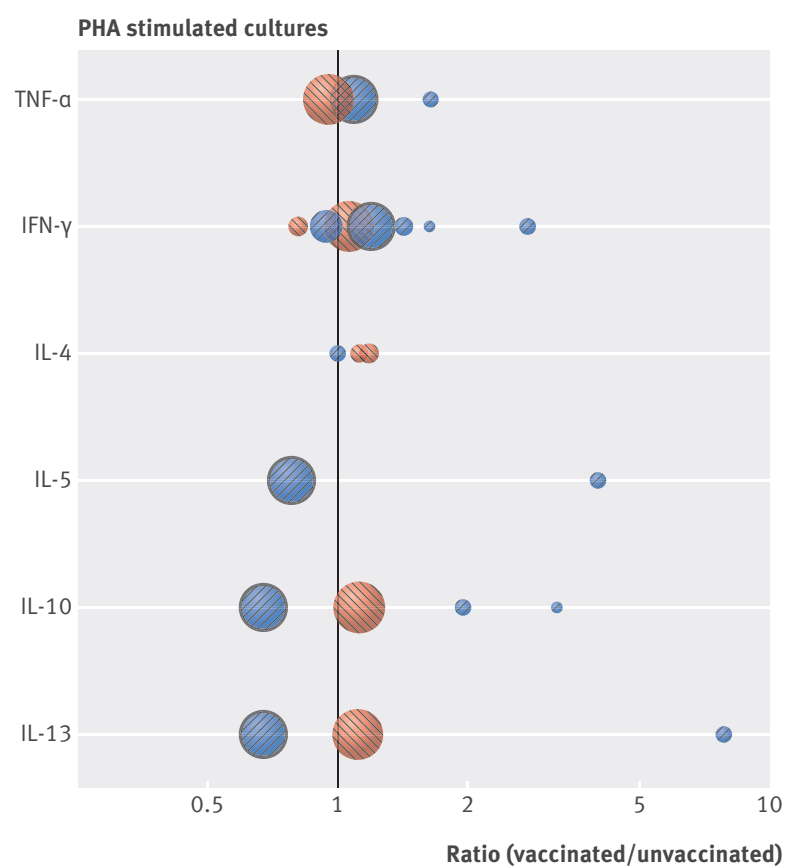

Lymphoproliferation and leukocyte counts

Total lymphocytes

Leukocytes

Neutrophils

Monocytes

CD14+

Eosinophils

CD8 count

CD4 count

$\gamma \delta+$

$a \beta+$

Proliferation (PHA)

$$
\mathrm{HBsAg}
$$

CD4 (tetanus)

CD8 (tetanus)

үס+ (tetanus)

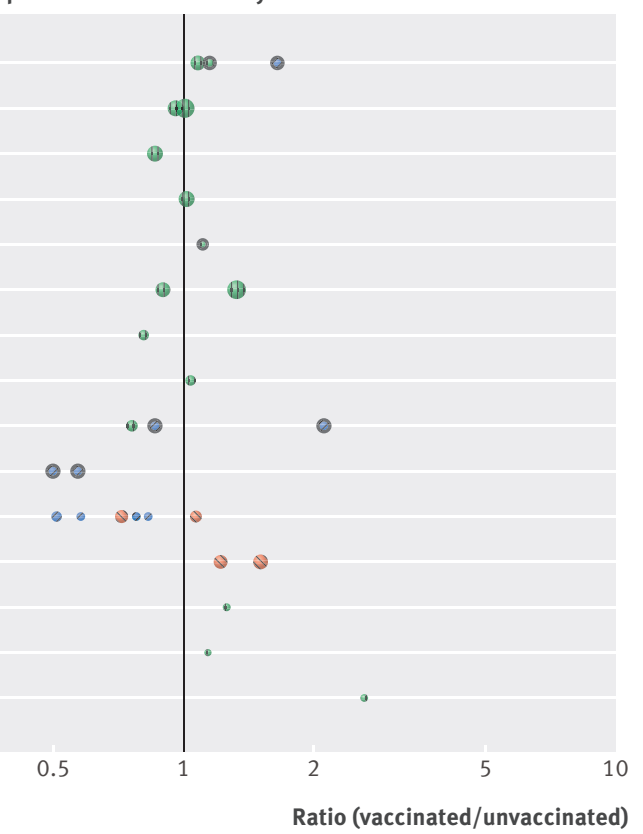

Fig 2 | Non-specific immunological responses to BCG vaccination for unstimulated cultures, PHA stimulated cultures, IFN- $\gamma$ responses, and lymphoproliferation and leukocyte counts from included BCG studies. Each circle represents responses from one study, calculated as either ratio of responses in those vaccinated compared with those unvaccinated (no black outer circle) or responses before vaccination compared with after vaccination (black outer circles). No estimate of variability is given and pooling of results was not justified because of inconsistency in summary statistics reported and poor reporting of results. Size of circles is proportionate to number of samples analysed. Ratios of geometric means (red circles with left hatching), ratios of medians (blue circles with right hatching), ratios of means (green circles with vertical hatching), and mean fold rise within individual (yellow circles)

appendix 4 provides detailed tabulated data for the fig). One study that compared responses between vaccinated groups showed an increase in IFN- $\gamma$, IL-2, and IL-10 in the vaccinated compared with the control group. ${ }^{35}$ The remaining responses to PHA were all from cohort studies and did not show a consistent direction of effect for any of the variables except for sIL-2Ra and $\beta 2$ microglobulin, which showed small relative increases after vaccination in relatively smaller cohort sizes. Cohort studies in which unstimulated assays were conducted showed decreases in IL-4, MIP-1 $\beta$, and sIL-2R $\alpha$ and no consistent direction of response for IFN- $\gamma$ (fig $\mathrm{G}$ in appendix 3). 
Ratios of geometric means $\bigcirc$ Ratios of medians 10 Ratios of means
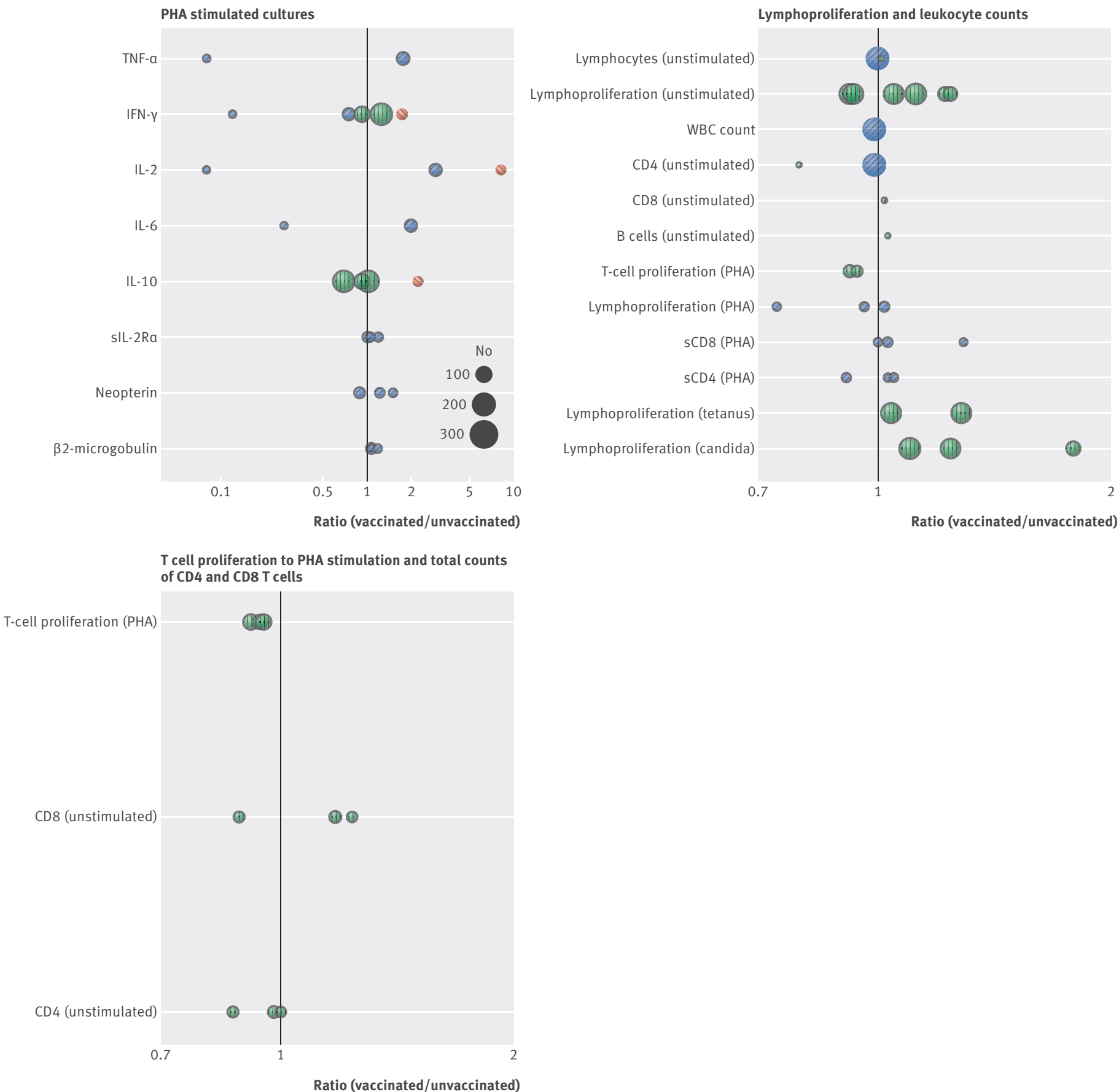

Fig 3 | Non-specific immunological responses to measles and MMR vaccination. PHA stimulated cultures, lymphoproliferation and leukocyte counts, and T cell proliferation to PHA stimulation and total counts of CD4 and CD8 T cells in included MMR vaccine studies. Each circle represents responses from one study, calculated as either ratio of responses in those vaccinated compared with those unvaccinated (no black outer circle) or responses before vaccination compared with after vaccination (black outer circles). No estimate of variability is given and pooling of results was not justified due to inconsistency in summary statistics reported and poor reporting of results. Size of circles is proportionate to number of samples analysed. Ratios of geometric means (red circles with left hatching), ratios of medians (blue circles with right hatching), and ratios of means (green circles with vertical hatching)

Lymphoproliferation was reported in response to tetanus toxoid, Candida species, and PHA stimulation as well as unstimulated assays (fig 3). In cohort studies reporting responses to $C$ albicans and tetanus toxoid, lymphoproliferation was consistently raised after vaccination. Total $\mathrm{T}$ cell proliferation was consistently reduced and soluble CD8 responses were consistently increased after PHA stimulation. One study that compared vaccinated with unvaccinated children showed overall values for ex vivo white blood cell counts and in vitro proliferative responses by unstimulated lymphocyte and CD4 T cells that lay close to the null. ${ }^{32}$ 


\section{MMR vaccine studies}

Overall data were extracted from three papers reporting responses to non-specific stimuli in MMR studies (table $\mathrm{C}$, appendix 2). Two papers conducted studies in children aged under 5 , while the third followed up vaccinated infants at mean age of 6.14 years. These papers reported 10 different immunological variables using five types of stimulants, resulting in 13 unique combinations.

CD4, and CD8 responses were the most commonly reported variables in cohort studies of T cell proliferation in response to PHA stimulation (fig 3). T cell proliferation in response to PHA stimulation was consistently reduced. Unstimulated CD4 responses also trended towards a reduction while unstimulated CD8 responses had no consistent direction of effect.

\section{Tetanus vaccine studies}

Ten studies reported responses to non-specific stimuli after tetanus vaccination (table D in appendix 2). They reported 21 different immunological variables (primarily lymphocyte proliferation and cytokines) and used 14 types of stimulants, resulting in 36 unique combinations. Only one study (by Borut and colleagues ${ }^{39}$ ) involved children aged under 5 , who made up only a fraction of the total study cohort.

Two studies reported cytokine production to mitogens after tetanus toxoid vaccination. After PHA stimulation, increases in IFN- $\gamma$ and IL-13 were noted in participants with non-allergic rhinitis compared with allergic rhinitis. ${ }^{41}$ One study included women randomised to palm oil or placebo and compared lymphoproliferative responses before and 56 days after vaccine receipt; stimulation with concanavalin A (ConA) resulted in higher IFN- $\gamma$ and IL-4 titres in both groups, while lipopolysaccharide generated higher IL-6 titres only in women who received palm oil..$^{45}$ Age seemed to play a role in blastogenesis to ConA and PHA after tetanus toxoid vaccination, with reduced responses in elderly adults compared with young adults. ${ }^{38}$ One study that examined skin test responses to tetanus toxoid noted that there was increased monocyte chemotaxis in those participants with positive skin test responses. ${ }^{39}$

\section{DTP, DT, and pertussis vaccine studies}

One study explored the effect of vitamin A on cytokine (IFN- $\gamma$, TNF- $\alpha$, IL-10, IL-5, and IL-13) responses in relation to receipt of DTP vaccination and noted no significant differences (fig $\mathrm{H}$ in appendix 3). ${ }^{49}$ This study, however, did report a significant decrease in monocyte count in the group not receiving vitamin A in conjunction with DTP. Interestingly, one DT (diphtheriatetanus) and one DTP study showed vaccine interference. The DT study showed increased herpes simplex virus and polio antibody titres, while the DTP study showed an increase in antibody to pneumococcus serotype 14 and polio neutralising responses.

We identified 10 studies that contained assays of non-specific immunological responses after immunisation with DTP or DT (table E in appendix 2). Notably, five studies reported to have or were likely to have coadministered a polio vaccine.
One study reported the comparison of a monovalent to trivalent pertussis vaccine in adults (table $\mathrm{F}$ in appendix 2). ${ }^{50}$ In this study culture stimulation indices to tetanus toxoid stimulation were measured but no statistical testing was performed.

\section{Discussion}

Principal findings

This is the first systematic review of non-specific immunological effects after human vaccination. Study designs were heterogeneous, and we could not carry out a meta-analysis. Included studies also had a low level of evidence quality. We could not conclude from the current available data that there are any consistent findings to confirm or discard the occurrence of non-specific immunological effects after vaccination with BCG, diphtheria, pertussis, tetanus, or measles containing vaccines. In addition, data from the included papers were not presented in a form that allowed us to assess the effect of sex on non-specific immunological effects. More meaningful conclusions might be drawn if raw data analyses could be conducted with unpublished and published data. If the same summary statistics could be computed for each study then meta-analysis might be possible, though there would still remain large diversity in study design, timing of assessments, and age at vaccination.

\section{Strengths and limitations}

Our review showed that a multitude of variables have been used to assess non-specific immunological effects of vaccines over the past six decades. Many of these are reported only once, and it is in these situations that single significant $P$ values need to be interpreted with caution. Stronger evidence for any effect can be found when more than one study has assessed the same variable and when confirmatory results can be found from different studies.

The improvement in technology for testing immunological variables (such as multiplex assays) allows multiple tests to be assessed at one time with one blood sample and greatly increases the chances of false positive results occurring because of chance alone. The standard arbitrary cut point used for significance testing in these situations $(\mathrm{P}<0.05)$ means that there is a $5 \%$ chance of a false positive result with every $P$ value computed. If a study reports the results of a multiplex assay testing multiple separate variables and each one is tested at the $\mathrm{P}<0.05$ level, then the chances that one of those variables will show a significant difference where none exists is high.

\section{Comparison with other studies}

Conceptually, we would expect heterogeneity of responses because the reported variables are singular measures of a complex biological system where a matrix of positive and negative responses by immunological variables can be seen. It is also important to consider potential confounders that could play a role-for example, contamination might explain some changes in unstimulated culture systems. Notably, this review 
shows some consistent patterns of effect that could be relevant in an immunological context. For example, in most studies that reported IFN- $\gamma$ responses in people vaccinated with BCG, there was an increase in production of the cytokine in unstimulated and stimulated in vitro conditions, both over time within a cohort and within groups that had received BCG compared with controls. The cytokine profile of PHA stimulated assays from BCG studies also showed a trend towards a pro-inflammatory response in cohort studies. Interestingly, the studies comparing vaccinated versus control groups indicate a more general increase in both pro-inflammatory and anti-inflammatory profiles. This suggests that the effect seen in the cohort studies could be ontogenetic in nature, consistent with in vitro studies of human blood stimulated with toll-like receptor agonists that show an increase in pro-inflammatory and decrease in anti-inflammatory cytokines during infancy. ${ }^{51}$

Three study cohorts assessed proliferative and IFN- $\gamma$ responses to HBsAg stimulation of peripheral blood mononuclear cells after BCG vaccination. ${ }^{2325}$ In two, IFN- $\gamma$ showed an increase in the respective variable for the vaccinated compared with the control group. ${ }^{25}$ Interestingly, responses to bacterial and fungal antigens in both BCG and measles vaccine studies showed increases in the respective variables measured. These findings are consistent with the results of animal studies that have shown acquired cellular resistance as a result of altered responsiveness of monocytes and macrophages..$^{52}$ This is supported by the trend towards an increase in monocyte responses in BCG vaccinated compared with control groups in this review. Furthermore, both animal and human studies support a role for activation of innate pattern recognition receptors triggering metabolic pathways and epigenetic changes that result in this response..$^{5322}$

\section{Conclusions and policy implications}

The lack of clear high quality evidence does not confirm or exclude the possibility of non-specific immunological effects after vaccination, which are well described in animal studies and accepted by many immunologists as occurring in humans. ${ }^{545}$ The human data, however, do not provide the necessary evidence to provide any confidence in the nature, quality, quantity, kinetics, or impact of non-specific immunological effects in young children after vaccination nor its translation into explaining morbidity or mortality outcomes.

Measurement of conventional immunological variables (such as antibody titres and cellular responses) provides little mechanistic insight into the relatedness of vaccination to an epidemiological outcome. Technological advances, however, mean that it might now be possible to design studies that can examine this issue systematically. For example, by using systems biology approaches, studies on yellow fever and influenza vaccination have uncovered some key processes that drive vaccine immune responses. ${ }^{5657}$ In addition, studies examining disease conditions (including infectious causes) have described the roles specific molecular pathways play in susceptibility. ${ }^{58}$ Thus systems biology provides an avenue for describing the biological networks that are perturbed by immunisation and how these can mechanistically relate to well defined epidemiological outcomes such as susceptibility to infectious disease. In addition, it has advantages in being able to incorporate complex considerations such as multiple vaccine antigens administered simultaneously. By examining how the molecular expression profiles generated after vaccination relate to those found at the time of a measured epidemiological outcome it would be possible to identify whether heterologous effects exist and, if so, how they exert their effects. Further validation would then be required perhaps in animal models or using in vitro systems.

Design of studies that would aid understanding of heterologous effects of vaccines should take account of four key considerations: identification of the main endpoints (morbidity or mortality) that will be used to assess the importance of any observed immunological effects; strategies to take account of the complexity of multiple antigens given at one time; the order in which vaccines are given in the immunisation schedule; and which biological measurements to use in the assessment. To appropriately examine these considerations it is necessary to undertake pilot mechanistic studies that would use new technological approaches to identify suitable laboratory variables to study in a larger epidemiological study with relevant endpoints. These studies would most readily be carried out in small cohorts of adults or animal based studies in which multiple sampling time points can be acquired, analysed with new high throughput technology such as standardised cellular phenotyping ${ }^{59}$ and multiplex serological assays coupled with the high dimensional molecular systems biology approaches to define these mechanisms.

Future detailed studies using a systems biology approach to capture the transcriptional, epigenetic, and immunological effects of vaccines could provide data on the timing, duration, quality, and magnitude of such effects. This would entail a rigorous statistical approach to correct for multiple testing. It is particularly important to gain an understanding of whether any such measurable effects are able to influence future inflammatory or innate/acquired immunological responses to exposure with vaccines or infectious agents. If reproducible signals are identified, these could be used in large scale studies with relevant epidemiological endpoints in children to characterise the clinical importance of such vaccine effects.

We acknowledge the support provided by WHO, Department of Immunization Vaccines and Biologicals, and all the members of the WHO SAGE working group on non-specific effects of vaccines. We also thank Henry Ebron and Peter O’Blenis for their support in using DistillerSR software.

Contributors: RK performed screening, selected articles for inclusions, assessed risk of bias, analysed data, and wrote the report. MV extracted and analysed the data. FMcQ performed screening, selected articles for inclusion, and assessed risk of bias. CS, DO'C, KdN, RR, OO, and UU performed screening and extracted data. AJP revised the protocol, performed screening, and adjudicated on conflicts on article inclusion and risk of bias assessment. All authors approved this version for publication. All authors, external and internal, had full access to all of the data (including statistical reports and tables) in the 
study and can take responsibility for the integrity of the data and the accuracy of the data analysis. RK and AJP are guarantors.

Funding: This work was supported by WHO. The work was commissioned as an independent evidence review by WHO. WHO staff provided advice about the topic area throughout the project and implemented the search of bibliographic databases.

Competing interests: All authors have completed the ICMJE uniform disclosure form at www.icmje.org/coi_disclosure.pdf (available on request from the corresponding author) and declare that AJP has previously conducted vaccine studies on behalf of Oxford University that were sponsored by manufacturers of vaccines but does not receive any personal payments from them. The University of Oxford has received unrestricted educational grants from vaccine manufacturers

Ethical approval: Not required.

Data sharing: No additional data available. A copy of the review protocol is available from the corresponding author.

Transparency: The lead author affirms that the manuscript is an honest, accurate, and transparent account of the study being reported that no important aspects of the study have been omitted; and that any discrepancies from the study as planned (and, if relevant, registered) have been explained.

This is an Open Access article distributed in accordance with the Creative Commons Attribution Non Commercial (CC BY-NC 3.0) license, which permits others to distribute, remix, adapt, build upon this work non-commercially, and license their derivative works on different terms, provided the original work is properly cited and the use is noncommercial. See: http://creativecommons.org/licenses/by-nc/3.0/.

1 Aaby P, Kollmann TR, Benn CS. Nonspecific effects of neonatal and infant vaccination: public-health, immunological and conceptual challenges. Nat Immunol 2014;15:895-9. doi:10.1038/ni.2961.

2 Benn CS, Netea MG, Selin LK, Aaby P. A small jab - a big effect: nonspecific immunomodulation by vaccines. Trends Immunol 2013;34:431-9. doi:10.1016/j.it.2013.04.004.

3 Aaby P, Benn C, Nielsen J, Lisse IM, Rodrigues A, Ravn H. Testing the hypothesis that diphtheria-tetanus-pertussis vaccine has negative non-specific and sex-differential effects on child survival in high-mortality countries. BMJ Open 2012;2:e000707. doi:10.1136/ bmjopen-2011-000707.

4 Aaby P, Ravn H, Roth A, et al. Early diphtheria-tetanus-pertussis vaccination associated with higher female mortality and no difference in male mortality in a cohort of low birthweight children: an observational study within a randomised trial. Arch Dis Child 2012;97:685-91. doi:10.1136/archdischild-2011-300646.

5 Vaugelade J, Pinchinat S, Guiella G, Elguero E, Simondon F. Non-specific effects of vaccination on child survival: prospective cohort study in Burkina Faso. BMJ 2004;329:1309. doi:10.1136/ bmj.38261.496366.82

6 Krishnan A, Srivastava R, Dwivedi P, Ng N, Byass P, Pandav CS. Non-specific sex-differential effect of DTP vaccination may partially explain the excess girl child mortality in Ballabgarh, India. Trop Med Int Health 2013;18:1329-37. doi:10.1111/tmi.12192.

7 Pollard AJ. Non-specific effects of vaccines: RCTs, not observational studies, are needed. Arch Dis Child 2012;97:677-8. doi:10.1136/ archdischild-2012-301873

8 Farrington CP, Firth MJ, Moulton LH, Ravn H, Andersen PK, Evans S. Working Group on Non-specific Effects of Vaccines. Epidemiological studies of the non-specific effects of vaccines: II--methodological issues in the design and analysis of cohort studies. Trop Med Int Health 2009:14:977-85. doi:10.1111/j.1365-3156.2009.02302x

9 Rehermann B, Shin E-C. Private aspects of heterologous immunity. J Exp Med 2005;201:667-70. doi:10.1084/jem.20050220.

10 Welsh RM, Fujinami RS. Pathogenic epitopes, heterologous immunity and vaccine design. Nat Rev Microbiol 2007;5:555-63. doi:10.1038/ nrmicro1709.

11 Sørup S, Benn CS, Stensballe LG, Aaby P, Ravn H. Measles-mumpsrubella vaccination and respiratory syncytial virus-associated hospital contact. Vaccine 2015;33:237-45. doi:10.1016/j.vaccine.2014.07.110.

12 Aaby P, Garly M-L, Nielsen J, et al. Increased female-male mortality ratio associated with inactivated polio and diphtheria-tetanuspertussis vaccines: Observations from vaccination trials in Guinea-Bissau. Pediatr Infect Dis / 2007;26:247-52. http://www.ncbi. nlm.nih.gov/pubmed/17484223. doi:10.1097/01. inf.0000256735.05098.01.

13 Yokomizo A, Kanimoto Y, Okamura T, et al. Randomized Controlled Study of the Efficacy, Safety and Quality of Life of Low-Dose Bacillus Calmette-Guerin Instillation Therapy for Non-Muscle-Invasive Bladder Cancer. J Urol 2015;195:41-6. doi:10.1016/j.juro.2015.08.075

14 Stewart JH 4th, Levine EA. Role of bacillus Calmette-Guérin in the treatment of advanced melanoma. Expert Rev Anticancer Ther 2011;11:1671-6. doi:10.1586/era.11.163.
15 Nofal A, Nofal E, Yosef A, Nofal H. Treatment of recalcitrant warts with intralesional measles, mumps, and rubella vaccine: a promising approach. Int J Dermatol 2015:54:667-71. doi:10.1111/ijd.12480.

16 WHO. SAGE Working Group on non-specific effects of vaccines (March-June 2013). http://www.who.int/immunization/sage/ sage wg non specific effects vaccines march2013/en/

17 WHO. Weekly Epidemiological Record 2008;83,32:285-292. http:// www.who.int/wer/2008/wer8332/en/

18 WHO. Weekly Epidemiological Record 2014;89,21:221-236. http:// www.who.int/wer/2014/wer8921/en/.

19 Burl S. The Role of Regulatory T cells in Early Life Immunity to BCG: Influence of Exposure to Environmental Mycobacteria.Open University, 2009.

20 Djuardi Y, Sartono E, Wibowo H, Supali T, Yazdanbakhsh M. A longitudinal study of BCG vaccination in early childhood: the development of innate and adaptive immune responses. PLoS One 2010;5:e14066. doi:10.1371/journal.pone.0014066.

21 Faustman DL, Wang L, Okubo Y, et al. Proof-of-concept, randomized, controlled clinical trial of Bacillus-Calmette-Guerin for treatment of long-term type 1 diabetes. PLoS One 2012;7:e41756. doi:10.1371/ journal.pone.0041756.

22 Kleinnijenhuis J, Quintin J, Preijers F, et al. Bacille Calmette-Guerin induces NOD2-dependent nonspecific protection from reinfection via epigenetic reprogramming of monocytes. Proc Natl Acad Sci U S A 2012;109:17537-42. doi:10.1073/pnas.1202870109.

23 Libraty DH, Zhang L, Woda M, et al. Neonatal BCG vaccination is associated with enhanced T-helper 1 immune responses to heterologous infant vaccines. Trials Vaccinol 2014;3:1-5. doi:10.1016/j.trivac.2013.11.004.

24 Marks GB, Ng K, Zhou J, et al. The effect of neonatal BCG vaccination on atopy and asthma at age 7 to 14 years: an historical cohort study in a community with a very low prevalence of tuberculosis infection and a high prevalence of atopic disease. J Allergy Clin Immunol 2003;111:541-9. doi:10.1067/mai.2003.171

25 Ota MO, Vekemans J, Schlegel-Haueter SE, et al. Influence of Mycobacterium bovis bacillus Calmette-Guérin on antibody and cytokine responses to human neonatal vaccination. J Immunol 2002;168:919-25. doi:10.4049/jimmunol.168.2.919.

26 Taştan Y, Arvas A, Demir G, Alikaşifoğlu M, Gür E, Kiray E. Influence of Bacillus Calmette-Guèrin vaccination at birth and 2 months old age on the peripheral blood T-cell subpopulations [gamma/delta and alpha-beta T cell]. Pediatr Allergy Immunol 2005;16:624-9. doi:10.1111/j.1399-3038.2005.00329.x.

27 Vargas MH, Bernal-Alcántara DA, Vaca MA, Franco-Marina F, Lascurain R. Effect of BCG vaccination in asthmatic schoolchildren. Pediatr Allergy Immunol 2004;15:415-20. doi:10.1111/j.13993038.2004.00198.x

28 Vijaya Lakshmi V, Kumar S, Surekha Rani H, Suman LG, Murthy KJ. Tuberculin specific T cell responses in BCG vaccinated children. Indian Pediatr 2005;42:36-40

29 Weir RE, Black GF, Dockrell HM, et al. Mycobacterial purified protein derivatives stimulate innate immunity: Malawians show enhanced tumor necrosis factor alpha, interleukin-1 beta (IL-1beta), and IL-10 responses compared to those of adolescents in the United Kingdom. Infect Immun 2004:72:1807-11. doi:10.1128/IAI.72.3.1807-1811.2004

30 Hennino A, Cornu C, Rozieres A, et al. Influence of measles vaccination on the progression of atopic dermatitis in infants. Pediatr Allergy Immunol 2007:18.385-90 doi:10.1111/j.1399-3038.2007.00537x.

31 Hussey GD, Goddard EA, Hughes J, et al. The effect of EdmonstonZagreb and Schwarz measles vaccines on immune response in infants. / Infect Dis 1996;173:1320-6. doi:10.1093/infdis/173.6.1320.

32 Lisse IM, Aaby P, Knudsen K, Whittle H, Andersen H. Long term impact of high titer Edmonston-Zagreb measles vaccine on T lymphocyte subsets. Pediatr Infect Dis / 1994;13:109-12. doi:10.1097/00006454-199402000-00006.

33 Ovsyannikova IG, Reid KC, Jacobson RM, Oberg AL, Klee GG, Poland GA. Cytokine production patterns and antibody response to measles vaccine. Vaccine 2003;21:3946-53. doi:10.1016/S0264-410X(03)00272-X.

34 Samb B, Whittle H, Aaby P, et al. No evidence of long-term immunosuppression after high-titer Edmonstron-Zagreb measles vaccination in Senegal. J Infect Dis 1995;171:506-8. doi:10.1093/ infdis/171.2.506.

35 Schnorr JJ, Cutts FT, Wheeler JG, et al. Immune modulation after measles vaccination of 6-9 months old Bangladeshi infants. Vaccine 2001;19:1503-10. doi:10.1016/S0264-410X(00)00349-2.

36 Pabst HF, Spady DW, Carson MM, Stelfox HT, Beeler JA, Krezolek MP. Kinetics of immunologic responses after primary MMR vaccination. Vaccine 1997;15:10-4. doi:10.1016/S0264-410X(96)00124-7.

37 Rager-Zisman B, Bazarsky E, Skibin A, et al. The effect of measlesmumps-rubella (MMR) immunization on the immune responses of previously immunized primary school children. Vaccine 2003:21:2580-8. doi:10.1016/S0264-410X(03)00053-7.

38 Armitage KB, Duffy EG, Mincek MA, et al. Transient normalization of lymphocyte blastogenic and specific antibody responses following boosting of healthy elderly subjects with tetanus toxoid. J Gerontol 1993;48:M19-25. doi:10.1093/geronj/48.1.M19. 
39 Borut TC, Ank BJ, Gard SE, Stiehm ER. Tetanus toxoid skin test in children: correlation with in vitro lymphocyte stimulation and monocyte chemotaxis. / Pediatr 1980;97:567-73. doi:10.1016/ S0022-3476(80)80010-2.

40 Chollet P, Chassagne J, Philippe P, et al. Peripheral lymphocytes changes in the anamnestic response to tetanus toxoid challenge. Clin Exp Immunol 1979;37:152-61.

41 Gentile D, Trecki J, Patel A, Fausnight T, Angelini B, Skoner D. Effect of tetanus immunization on t-helper cytokine production in adults with and without allergic rhinitis. Allergy Asthma Proc 2006;27:197-201. doi:10.2500/aap.2006.27.2857.

42 Mahalingam D, Radhakrishnan AK, Amom Z, Ibrahim N, Nesaretnam K. Effects of supplementation with tocotrienol-rich fraction on immune response to tetanus toxoid immunization in normal healthy volunteers. Eur J Clin Nutr 2011;65:63-9. doi:10.1038/ejcn.2010.184.

43 Fernandes JR, Wasserman S, Snider DP. Stimulation of anti-polio and anti-HSV IgA pre-plasma cell response in blood following parenteral immunization with tetanus-diphtheria vaccine. Vaccine 2010;28:1493-8. doi:10.1016/j.vaccine.2009.11.057.

44 Halasa NB, O'Shea A, Shi IR, LaFleur BJ, Edwards KM. Poor immun responses to a birth dose of diphtheria, tetanus, and acellular pertussis vaccine. J Pediatr 2008;153:327-32. doi:10.1016/j.jpeds.2008.03.011.

45 Heine G, Drozdenko G, Lahl A, et al. Efficient tetanus toxoid immunization on vitamin D supplementation. Eur J Clin Nutr 2011;65:329-34. doi:10.1038/ejcn.2010.276

46 Rowe J, Macaubas C, Monger TM, et al. Antigen-specific responses to diphtheria-tetanus-acellular pertussis vaccine in human infants are initially Th2 polarized. Infect Immun 2000;68:3873-7. doi:10.1128/ IAl.68.7.3873-3877.2000.

47 El Yousfi M, Mercier S, Breuillé D, et al. The inflammatory response to vaccination is altered in the elderly. Mech Ageing Dev 2005;126:874 81. doi:10.1016/j.mad.2005.03.008

48 Hoft DF, Brown RM, Roodman ST. Bacille Calmette-Guérin vaccination enhances human gamma delta T cell responsiveness to mycobacteria suggestive of a memory-like phenotype. J Immunol 1998;161:1045-54.

49 Jorgensen MJ, Fisker AB, Sartono E, et al. The effect of at-birth vitamin A supplementation on differential leucocyte counts and in vitro cytokine production: an immunological study nested within a randomised trial in Guinea-Bissau. Br J Nutr 2013;109:467-77. doi:10.1017/S0007114512001304.
50 Di Tommaso A, Bartalini M, Peppoloni S, Podda A, Rappuoli R, De Magistris MT. Acellular pertussis vaccines containing genetically detoxified pertussis toxin induce long-lasting humoral and cellular responses in adults. Vaccine 1997;15:1218-24. doi:10.1016/S0264-410X(97)00023-6.

51 Kollmann TR, Levy O, Montgomery RR, Goriely S. Innate immune function by Toll-like receptors: distinct responses in newborns and the elderly. Immunity 2012;37:771-83. doi:10.1016/j.immuni.2012.10.014.

52 MacKaness GB. The Immunological Basis of Acquired Cellular Resistance. J Exp Med 1964:120:105-20. doi:10.1084/jem.120.1.105.

53 Saeed S, Quintin J, Kerstens HH, et al. Epigenetic programming of monocyte-to-macrophage differentiation and trained innate immunity. Science 2014;345:1251086. doi:10.1126/science.1251086.

54 Choi IS, Lin XH, Koh YA, Koh Yl, Lee HC. Strain-dependent suppressive effects of BCG vaccination on asthmatic reactions in BALB/c mice. Ann Allergy Asthma Immunol 2005;95:571-8. doi:10.1016/ S1081-1206(10)61021-6.

55 Parra M, Liu X, Derrick SC, et al. Molecular analysis of non-specific protection against murine malaria induced by BCG vaccination. PLoS One 2013;8:e66115. doi:10.1371/journal.pone.0066115.

56 Nakaya HI, Wrammert J, Lee EK, et al. Systems biology of vaccination for seasonal influenza in humans. Nat Immunol 2011;12:786-95. doi:10.1038/ni.2067

57 Gaucher D, Therrien R, Kettaf N, et al. Yellow fever vaccine induces integrated multilineage and polyfunctional immune responses. J Exp Med 2008;205:3119-31. doi:10.1084/jem.20082292

58 Mejias A, Ramilo 0. Transcriptional profiling in infectious diseases: ready for prime time? J Infect 2014;68(Suppl 1):S94-9. doi:10.1016/j. jinf.2013.09.018.

59 Kalina T, Flores-Montero J, Lecrevisse Q, et al. Quality assessment program for EuroFlow protocols: summary results of four-year (2010-2013) quality assurance rounds. Cytometry A 2015;87:145-56. doi:10.1002/cyto.a.22581.

Appendix 1: Search terms

Appendix 2: Supplementary tables A-L and references Appendix 3: Supplementary figures with tabulated data Appendix 4: Detailed tabulated data for figs 2 and 3 